Farum

Sociológico

\section{Forum Sociológico}

Série II

$24 \mid 2014$

Circulação de saberes e desafios em saúde

\title{
Saúde, participação e abordagens deliberativas. Potencialidades e limites dos júris de cidadãos
}

Health, participation and deliberative approaches. Potential and limits of citizens' juries

Mauro Serapioni

\section{CpenEdition}

Journals

Edição electrónica

URL: https://journals.openedition.org/sociologico/1101

DOI: 10.4000/sociologico.1101

ISSN: 2182-7427

Editora

CICS.NOVA - Centro Interdisciplinar de Ciências Sociais da Universidade Nova de Lisboa

Edição impressa

Paginação: 95-103

ISSN: 0872-8380

Refêrencia eletrónica

Mauro Serapioni, «Saúde, participação e abordagens deliberativas. Potencialidades e limites dos júris de cidadãos», Forum Sociológico [Online], 24 | 2014, posto online no dia 01 novembro 2014, consultado o 29 março 2022. URL: http://journals.openedition.org/sociologico/1101 ; DOl: https://doi.org/ 10.4000/sociologico.1101

Este documento foi criado de forma automática no dia 29 março 2022.

(c) CICS.NOVA 


\section{Saúde, participação e abordagens deliberativas. Potencialidades e limites dos júris de cidadãos}

Health, participation and deliberative approaches. Potential and limits of citizens' juries

\section{Mauro Serapioni}

\section{Introdução}

1 Nos últimos vinte anos, têm sido publicadas muitas análises e ensaios com o objetivo de reformular a teoria e prática da democracia à luz da viragem deliberativa. Uma vasta $\mathrm{e}$ crescente literatura (tanto teórica como empírica) e vários académicos têm definido a deliberação pública como pedra angular da democracia participativa e do governo representativo (Fishkin, 1995; Elster, 1998; Chambers, 2003; Gastil, 2000; Dryzek, 2000; Pateman, 2012). Desde os finais da década de 1990, a deliberação é considerada essencial para o desenvolvimento da democracia e da teoria da participação dos cidadãos. Muitos autores revelaram-se cada vez mais interessados pela ideia de construir espaços públicos no seio da sociedade civil como locus de democratização da vida quotidiana (Taylor, 1989).

2 Recentemente, na perspetiva da governação, os métodos deliberativos receberam muita atenção enquanto estratégia inovadora para maximizar a interação entre os decisores e os cidadãos. Por exemplo, na área das políticas públicas, entre as décadas de $1980 \mathrm{e}$ 1990, assistiu-se a uma significativa mudança de uma abordagem baseada numa ciência política, centrada nos especialistas para a inclusão da voz dos cidadãos no debate político.

3 Os métodos deliberativos representam uma forma de participação que oferece aos indivíduos a oportunidade de expressarem os seus pontos de vista, aprenderem e compreenderem as posições dos outros participantes, identificar problemas e preferências em comum e terem uma ideia correta sobre as questões de importância 
pública. A prática deliberativa envolve, necessariamente, um processo coletivo de tomada de decisão, no qual os participantes têm a oportunidade de convergir numa visão partilhada, dando voz e relevância a todos os argumentos apresentados. No entanto, o consenso não tem de ser o derradeiro objetivo de um processo deliberativo, tal como afirmam Fung e Wright (2001: 19): "O aspeto importante da deliberação genuína é que os participantes encontram razões que podem aceitar em ações coletivas, não sendo necessário que apoiem totalmente a ação ou a considerem idealmente vantajosa."

Entre os benefícios dos processos deliberativos assinalados pela literatura (Abelson et al., 2003a; Scutchfield et al., 2006; Delli Carpini et al., 2004; Mitton et al., 2009; Gastil, 2010), destacam-se os seguintes: a capacidade de aumentar o conhecimento e a compreensão dos cidadãos; o potencial de alterar as opiniões dos participantes; a capacidade de aumentar o nível de tolerância e compreensão entre grupos ao aceitarem pontos de vista diferentes; um mecanismo qualificado para produzir decisões coletivas e aumentar a legitimidade das decisões; o potencial de restaurar a perda de confiança nas instituições públicas; um julgamento mais informado e refletido; o aumento da participação em ações políticas; e o reforço dos hábitos de participação cívica. Lehoux et al. (2009) recomendam a adoção de processos deliberativos devido ao seu potencial para maximizar a aprendizagem mútua, tanto entre especialistas como entre estes e não especialistas.

5 Este debate sobre governação, abordagens deliberativas e participação está ativamente presente no sistema de saúde. De facto, um dos aspetos principais das reformas na saúde dos últimos vinte anos tem sido a crescente importância atribuída à voz e perspetiva dos cidadãos. Muitos académicos têm realçado que a integração do conhecimento e da experiência dos doentes melhora a qualidade das decisões.

6 Este artigo irá fazer uma breve apresentação do debate sobre a importância da participação pública no setor da saúde e os principais métodos deliberativos adotados nesta área para envolver os cidadãos. De seguida, serão descritos os júris de cidadãos, destacando as respetivas características, capacidades e fragilidades através de uma revisão da literatura. De facto, apesar do amplo reconhecimento do seu potencial para melhorar a legitimidade, a responsabilização e os processos de tomada de decisão, também é importante identificar alguns aspetos cruciais que emergiram da análise da literatura. Para concluir, será abordada a questão fundamental da ausência de uma avaliação dos júris de cidadãos, assim como de outros esquemas deliberativos utilizados nas políticas públicas.

\section{Participação deliberativa no sistema de saúde}

7 Ao revermos a literatura internacional sobre participação na saúde, é possível observar que existe um consenso generalizado sobre a importância do envolvimento dos cidadãos nas decisões que afetam a sua saúde. Ao longo da última década, o debate participativo tem realçado a necessidade de novas abordagens para envolver os cidadãos numa interação mais ativa com os decisores. De acordo com Abelson (2003a: 240), esta nova tendência resultou, parcialmente, "da filosofia consumista neoliberal que dominou as décadas de 1980 e 1990 e de uma filosofia de governação que fomenta obrigações recíprocas entre cidadãos e governos e destaca a relevância da participação para fins coletivos e não individuais". 
8 A perspetiva deliberativa está a atrair um grande interesse no setor da saúde, onde existe uma crescente compreensão da necessidade de criar uma "esfera pública" adequada (Habermas, 1992), para encorajar o diálogo entre os vários atores no sistema de saúde. Em anos recentes, esta perspetiva tem recebido um maior destaque, graças à influência do trabalho de Habermas (1997), especialmente os conceitos de "situação discursiva ideal". Deste modo, os diferentes métodos deliberativos têm sido amplamente utilizados para envolver os cidadãos em discussões baseadas em valores, no processo de tomada de decisão, na definição de prioridades e na identificação das prioridades de investigação no sistema de saúde. Entre os métodos mais comuns utilizados para envolver os cidadãos no sistema de saúde, destacam-se os seguintes: júris de cidadãos, painéis de cidadãos, conferências de consenso, oficina de cidadãos, sondagens deliberativas e grupos de discussão deliberativa. Todos eles têm em comum o elemento deliberativo que inclui alguns aspetos essenciais, tais como: fornecer informações sobre o assunto em discussão a todos os participantes; descrever e formular os tópicos a serem analisados na linguagem do cidadão comum; considerar cuidadosamente as opiniões dos restantes participantes; e estimular a discussão para obter um consenso ou aproximar várias posições.

9 O reconhecimento dos limites dos métodos convencionais e a necessidade de obter visões aprofundadas sobre questões de saúde complexas influenciaram a procura de novos mecanismos para envolver os cidadãos. Deste modo, os processos deliberativos foram considerados adequados para estimular o debate, otimizar o conhecimento dos participantes e obter consenso em torno de valores públicos e sobre as prioridades dos serviços de saúde. Existe no setor da saúde uma crença generalizada de que os mecanismos deliberativos possuem um efeito salutar nos participantes e no sistema de saúde. Mas será isto verdade? O que nos diz a investigação empírica? Para responder a estas questões, foi realizada uma revisão da literatura (principalmente em materiais de língua inglesa) para analisar as experiências de participação através dos júris de cidadãos - um dos métodos deliberativos mais habitualmente utilizados nos sistemas de saúde. A análise, abrangendo o período entre 1996 e 2011, baseou-se em 46 trabalhos publicados: livros, capítulos de livros e artigos de revistas científicas.

\section{Os júris de cidadãos: origem e elementos-chave}

Os júris de cidadãos (JC) foram desenvolvidos de forma independente na década de 1970 nos Estados Unidos e na Alemanha (onde assumiram um formato ligeiramente diferente, sendo conhecidos como "células de planeamento") nas áreas da telecomunicação, agricultura e ambiente. Em seguida, durante a década de 1990, foram desenvolvidos no Reino Unido, Canadá, Austrália e Nova Zelândia. Ao longo das últimas duas décadas, o recurso a este dispositivo alastrou-se para muitos outros países (Espanha, Itália, Japão, Taiwan, etc.). Foram introduzidos no sistema de saúde britânico em 1994 pela Universidade de Birmingham e foram testados pelos investigadores do Instituto de Investigação em Políticas Públicas e da fundação King's Fund em Londres (Coote e Lenaghan, 1997; Parkinson, 2004). Inicialmente, os JC eram utilizados para ouvir as opiniões dos utentes de serviços de saúde mental (McIver, 1998), para atribuir financiamento aos serviços de saúde locais (Lenaghan et al. 1996), e para prevenir possíveis tensões resultantes de problemas de acesso às estruturas de saúde (Dale et al., 1998). Posteriormente, ganharam popularidade ao abordarem questões associadas ao 
racionamento dos cuidados de saúde, a definição de prioridades e a avaliação das tecnologias de saúde.

Os JC são constituídos por cerca de 12 a 20 membros do público, selecionados por um painel de especialistas em amostragem, em representação da população local. A seleção dos jurados é um processo muito delicado pois a amostra tem de ser representativa da população local e das diversas opiniões sobre o tópico em discussão. 0 grupo reúne durante quatro ou cinco dias para discutir, analisar e deliberar sobre assuntos relacionados com a saúde da comunidade local. Antes da reunião, os jurados são informados sobre os assuntos específicos sobre os quais lhes é pedido que deem uma opinião. Durante a sessão de trabalho, são providenciadas aos jurados informações e análises adicionais por parte de testemunhas previamente identificadas. As testemunhas são, normalmente, administradores, médicos especialistas, médicos de família, enfermeiros e até doentes com uma experiência relevante para o tema abordado. 0 trabalho do júri é "gerido" por dois moderadores. O júri analisa o material, faz perguntas às testemunhas e discute os vários aspetos do problema em pequenos grupos. Seguidamente, as suas conclusões são compiladas num relatório elaborado pelos dois moderadores, que é enviado a todos os jurados para aprovação antes de ser entregue à entidade de saúde. Os resultados, que podem obter ou não o consentimento unânime de todos os jurados, incluem recomendações operacionais que, apesar de não terem um caráter vinculativo, o administrador do distrito de saúde poderá ter em conta. A existência de um grupo diretor é essencial para coordenar os diferentes atores envolvidos nas várias etapas do processo: determinar as questões, preparar a agenda, identificar testemunhas, preparar as informações para os júris e elaborar o relatório final.

Os resultados das avaliações dos JC desenvolvidas no setor da saúde revelam diversos pontos positivos e virtudes, mas também apresentam alguns aspetos críticos (New, 1998; Serapioni, 2002; Gooberman-Hill et al., 2008). As virtudes dos JC, tal como outros métodos deliberativos, opõem-se a outras características fundamentais da participação democrática como, por exemplo, a representação, a influência e a qualidade das informações.

\section{Potencialidades e pontos positivos dos júris de cidadãos}

Muitos estudos comprovaram que podem ser retiradas diversas lições dos júris de cidadãos, realçando a capacidade de desenvolver uma opinião informada e novas perspetivas de análise sobre as principais questões de saúde relacionadas com as comunidades. A adoção da estratégia dos JC pode ter um papel importante na formulação de políticas sociais e de saúde pública ao nível local. De acordo com vários académicos, os JC desenvolveram uma forma interessante de descobrir valores e preferências da comunidade sobre questões de saúde, ao mesmo tempo que providenciam, também, uma plataforma adequada para os participantes expressarem os seus problemas e as suas prioridades, influenciando, assim, os serviços que lhes são prestados. Havendo tempo e recursos suficientes para realizar o JC, e se o processo de construção de conhecimentos e deliberação for bem desenvolvido, os jurados leigos conseguem absorver informações complexas, participar em discussões sensíveis e fazer 
escolhas sensatas. A Tabela 1 resume os benefícios mencionados dos júris de cidadãos compilados pela análise da literatura.

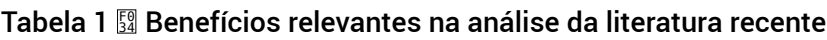

\begin{tabular}{|c|c|}
\hline $\begin{array}{l}\text { Os JC são uma forma de introduzir uma maior democracia na tomada de } \\
\text { decisões relativas ao sistema de saúde. }\end{array}$ & $\begin{array}{l}\text { Mooney e Blackwell, } \\
\text { 2004. Perth, Austrália. }\end{array}$ \\
\hline $\begin{array}{l}\text { Os resultados dos JC poderão ter um papel importante na formulação de } \\
\text { políticas sociais e de saúde pública. }\end{array}$ & $\begin{array}{l}\text { Elwood e Longley, } 2010 . \\
\text { Pontypridd, Rei- } \\
\text { no Unido. }\end{array}$ \\
\hline $\begin{array}{l}\text { O resultado dos JC sugere que um público informado não coloca a } \\
\text { privacidade pessoal acima dos benefícios societais. }\end{array}$ & $\begin{array}{l}\text { Parkin e Paul, } 2011 . \\
\text { Dunedin, Nova Ze- } \\
\text { lândia. }\end{array}$ \\
\hline $\begin{array}{l}\text { Os JC usufruem do debate e levam as suas responsabilidades } \\
\text { extremamente asério. }\end{array}$ & $\begin{array}{l}\text { Delap, 2001. Escócia, } \\
\text { Reino Unido. }\end{array}$ \\
\hline Os JC podem apresentar ideias de investigação e dar-lhes prioridade. & $\begin{array}{ll}\text { Herbison et al., } & 2009 . \\
\text { Dunedin, } & \text { Nova } \\
\text { Zelândia. } & \end{array}$ \\
\hline $\begin{array}{l}\text { Os JC proporcionam uma abordagem viável para envolver o público na } \\
\text { definição de prioridades. }\end{array}$ & $\begin{array}{l}\text { Menon e Stafinsky, } \\
2008 . \\
\text { Canadá. }\end{array}$ \\
\hline $\begin{array}{l}\text { Os JC demonstraram uma capacidade de assimilar informações, avaliar } \\
\text { criticamente as evidências e deliberar de forma sofisticada. }\end{array}$ & $\begin{array}{l}\text { Bennett e Smith, } 2007 . \\
\text { Edimburgo, Rei- } \\
\text { no Unido. }\end{array}$ \\
\hline $\begin{array}{l}\text { Os JC oferecem uma grande oportunidade para a disseminação de } \\
\text { informações e deliberação. }\end{array}$ & $\begin{array}{lll}\text { Paul et al., } & 2008 . \\
\text { Dunedin, } & & \text { Nova } \\
\text { Zelândia. } & \end{array}$ \\
\hline Os JC podem mudar a opinião dos participantes. & $\begin{array}{l}\text { Abelson et al., 2003a. } \\
\text { Canadá. }\end{array}$ \\
\hline $\begin{array}{l}\text { Os JC construíram mecanismos para assegurar que as opiniões dos } \\
\text { cidadãos têm influência sobre os serviços. }\end{array}$ & $\begin{array}{l}\text { Lenaghan, 1999. Reino } \\
\text { Unido. }\end{array}$ \\
\hline $\begin{array}{l}\text { Os JC oferecem perspetivas importantes sobre o modo como a deliberação } \\
\text { democrática pode ser institucionalizada nos processos contemporâneos } \\
\text { de tomada de decisões políticas. }\end{array}$ & $\begin{array}{l}\text { Smith e Wales, } 2000 . \\
\text { Reino Unido. }\end{array}$ \\
\hline Os JC permitem tomar decisões informadas e responsáveis. & $\begin{array}{l}\text { Culyer e Lomas, } 2006 . \\
\text { Canadá. }\end{array}$ \\
\hline
\end{tabular}

FONTE: elaboração própria com base na análise da literatura.

14 O método de envolvimento público adotado pelos júris de cidadãos representa outra virtude. Muitos académicos acreditam que os júris de cidadãos são "superiores" a 
outros métodos utilizados na área da saúde (inquéritos, grupos de discussão, conferências, etc.), devido à sua capacidade de estimular a reflexão dos jurados sobre os problemas em avaliação e por terem o objetivo de elaborar recomendações para serem submetidas à administração (Paul et al., 2008; Bennett e Smith, 2007; Iredale e Longley, 2007; Wakeford, 2002; McIver, 1998; Coote e Lenaghan, 1997). A "opinião informada" dos jurados é reconhecida como um dos principais motivos para as autoridades de saúde promoverem esta nova estratégia de envolvimento de cidadãos (New, 1998). Os jurados não só identificam os pontos críticos do sistema de saúde, tal como é prática comum em outros métodos, mas também refletem sobre os problemas que lhes foram remetidos e sugerem recomendações (Abelson et al., 2003a). A visão otimista sobre o potencial dos JC é visível na opinião do administrador de uma entidade de saúde, relatada por McIver (1998: 41): "É o Rolls-Royce dos métodos. Os outros são Minis."

\section{Desafios e pontos críticos dos júris de cidadãos}

Apesar do amplo reconhecimento do potencial dos júris de cidadãos para aumentar a legitimidade, responsabilização e melhorar o processo de tomada de decisões, também é importante identificar alguns aspetos críticos que emergiram das análises, tais como: a influência limitada sobre os decisores; o problema da representatividade; o nível de institucionalização; a questão do poder e da qualidade das informações; e o custo excessivo de realizar um júri de cidadãos.

\subsection{Influência limitada}

Tal como mencionado em seç̧ões anteriores, um princípio importante da abordagem deliberativa consiste no pressuposto de que os jurados possuem o conhecimento e a competência para alcançar argumentos fundamentados e conclusões informadas. Contudo, outro princípio-chave do exercício da democracia deliberativa é a capacidade de produzir recomendações e exercer algum tipo de influência sobre os decisores políticos. Por outras palavras, a eficácia de um mecanismo de participação deliberativa deve ser avaliada, em certa medida, pelo resultado alcançado. No entanto, tal como evidenciado por alguns autores (Pickard, 1998; New, 1998; Price, 2000; Iredale e Longley, 2007; Gooberman-Hill et al., 2008), não há garantias de que as recomendações do JC são consideradas pelas autoridades de saúde. Na verdade, os jurados apresentam um relatório final às autoridades de saúde, mas não participam nas discussões subsequentes e nos processos de tomada de decisão. Em alguns casos, segundo Price (2000: 275), diz-se que os JC tomam decisões mas isto apenas acontece em algumas circunstâncias: "Muitas vezes, os sentimentos e pensamentos dos jurados são relatados como se fossem decisões (...). No entanto, as decisões do júri não são conclusões." Tal como afirma Armour (1995: 186), isto significa que o modelo do JC, assim como outros modelos de participação, transforma os utentes em espetadores, externos à arena política, enquanto aguardam que as suas sugestões sejam incorporadas em processos de tomada de decisão.

17 O efeito limitado dos JC nas políticas locais também se destacou na experiência espanhola na área do planeamento urbano e da utilização do espaço público. De acordo com Font e Blanco (2007: 581), "uma das questões levantadas mais frequentemente por cidadãos quando lhes é pedido para participar é se as suas escolhas se irão traduzir em 
verdadeiras decisões". A este respeito, Blondiaux e Sintomer (2002: 31) colocam algumas perguntas: "Porquê participar na deliberação se não há perspetivas de ação?", "Como é possível convencer as pessoas a participar sem dar a garantia de que as suas opiniões serão úteis?". Uma preocupação comum foi destacada por Fung e Wright (2001), que afirmam que, mesmo que os aspetos processuais e os princípios das abordagens deliberativas representem um valor independente e desejável, os académicos e os profissionais irão avaliar estes exercícios participativos não só pela qualidade dos respetivos processos como também pelo impacto nos participantes e nos processos de tomada de decisão.

\subsection{Problema de representatividade}

18 O envolvimento de pequenos grupos de participantes como representantes do público em geral sujeita os processos deliberativos a fortes críticas. Têm sido levantadas questões sobre a medida em que as opiniões e perspetivas individuais expressadas nos júris de cidadãos são representativas das visões do público em geral (Gooberman-Hill et al., 2008). Podem 16 pessoas, escolhidas aleatoriamente e envolvidas em 3-4 dias de informação e deliberação intensas, representar a grande variedade das opiniões ao nível local ou regional? Deste modo, a abordagem deliberativa enfrenta a mesma lacuna de representatividade registada nos exercícios de participação baseados nos métodos convencionais, tal como é evidenciado por investigações desenvolvidas na década de 1990 (Zakus e Lysack, 1998; Lomas, 1997; Crouch, 1999). Apesar de serem recomendadas estratégias adequadas para atingir uma amostragem representativa, segundo Gooberman-Hill et al. (2008), isto incorre no "risco de excluir vozes sub-representadas". Neste sentido, vários estudos realçam o paradoxo dos métodos deliberativos que podem contribuir para o aumento das desigualdades na saúde pois, normalmente, os grupos sociais vulneráveis (imigrantes, idosos, pessoas com problemas de saúde mental, etc.) participam em menor grau do que os grupos com um maior nível de instrução e socialmente integrados. A mesma preocupação foi expressada por Fung e Wright (2001: 34):

"Um facto lamentável de todas as democracias contemporâneas é que os cidadãos mais favorecidos em termos de riqueza, educação, remuneração ou inclusão num grupo racial ou étnico dominante participam mais frequentemente e de forma mais eficaz do que os cidadãos mais desfavorecidos. As experiências [deliberativas] exigem formas intensivas de participação política que poderão agravar ainda mais estes enviesamentos da participação (...)."

19 Segundo Timotijevic e Raats (2007: 317), a técnica de recrutamento não foi capaz de "penetrar em comunidades excluídas (...) apesar de haver evidências de que a amostragem intencional através de organizações locais" representa uma "forma eficaz de recrutar grupos sociais difíceis de alcançar". Por este motivo, são recomendados métodos complementares para assegurar uma representação adequada de grupos marginalizados (Wakeford, 2002; Serapioni e Duxbury, 2012).

\subsection{Nível de institucionalização}

Outra fragilidade dos JC consiste no respetivo baixo nível de institucionalização no sistema de saúde. Os JC não são, de modo geral, reconhecidos nas regulamentações regionais ou nacionais e não atuam, rotineiramente, em todas as entidades de saúde. 
Além disso, em muitos casos, tal como evidenciado por alguns estudiosos (Pickard, 1998; Kashefi e Mort, 2004; Font e Blanco, 2007), os JC representam uma experiência isolada num contexto com poucas oportunidades de participação. A este respeito, poderão, certamente, representar um exercício eficaz de participação, mas apenas se forem integrados no esquema global da representação pública (Smith e Wales, 2000; Pateman, 2012).

21 A abordagem participativa institucionalizada, tal como realçado por vários autores (Abelson et al., 2003b; Mitton et al., 2009) parece ser uma estratégia promissora para envolver cidadãos e promover uma relação mais sustentável com os doentes e as associações de utentes. Esta é a melhor forma de desenvolver uma estratégia mais avançada de parceria e cidadania na saúde. Adicionalmente, estas estratégias evitam que a participação se torne um exercício esporádico que apenas é implementado mediante a iniciativa das autoridades de saúde, tal como foi o caso de várias metodologias deliberativas ocorridas nos sistemas de saúde ao longo dos últimos anos.

\subsection{Desequilíbrio do poder e qualidade da informação}

A deliberação, enquanto processo social, depende da persuasão para induzir os participantes a mudarem as suas opiniões e avaliações durante o processo de interação. Neste sentido, é totalmente distinto de outras formas de comunicação, incluindo a coerção, a manipulação ou o engano (Dryzek, 2000). No entanto, o pressuposto de que o poder pode ser excluído do diálogo deliberativo e de que as desigualdades entre os participantes podem ser reduzidas tem sido desafiado pela literatura, que destaca a centralidade das relações de poder. De facto, alguns estudos demonstraram que a centralidade do poder não diminuiu (Abelson et al., 2003b) e que os participantes interagem e confrontam-se entre si em situações e contextos caracterizados por disparidades de poder (Fung e Wright, 2001). Estas desigualdades derivam tanto de diferenças materiais e sociais entre os participantes que estão, aparentemente, em pé de igualdade, como de disparidades no nível de informação e conhecimento entre peritos e leigos. Referindo-se à experiência do júri de cidadãos de Bristol, GoobermanHill et al. (2008) demonstraram a existência de uma evidente assimetria de poder entre os patrocinadores (que detêm a informação) do processo deliberativo e os jurados.

23 A mesma preocupação foi expressada por Dunkerley e Glasner (1998: 188), que relataram as críticas de uma testemunha leiga após ter participado num júri de cidadãos sobre novas tecnologias genéticas: "Gostaria de ter sido ouvida de igual para igual com os profissionais, dispondo do mesmo tempo e da mesma oportunidade de fazer perguntas." Para Abelson et al. (2003a: 246), o desequilíbrio de poder é visível entre os próprios participantes que estão, aparentemente, a participar em pé de igualdade no processo deliberativo.

24 A questão da qualidade da informação disponibilizada aos jurados está estreitamente relacionada com o desequilíbrio de poder, pois estes podem ser influenciados pelos patrocinadores, organizadores e testemunhas. Aqui, têm sido evidenciados dois aspetos: (1) a possibilidade de avaliar a adequação e qualidade da informação fornecida aos jurados (Abelson et al., 2003b; Deng e Wu, 2010); e (2) a quantidade de informações apresentada aos jurados e a sua capacidade de ler e analisá-la no tempo atribuído. Vários autores demonstraram as dificuldades enfrentadas pelos jurados devido à amplitude das questões e a complexidade dos assuntos específicos (Lenaghan et al., 
1996; Gooberman-Hill et al., 2008). Outros investigadores destacaram o potencial de enviesamento que poderá influenciar as respostas dos jurados. Por exemplo, Glasner (2001: 43) realça o papel do grupo diretor na decisão sobre "quais as informações preliminares disponibilizadas aos jurados". Iredale e Longley (2007: 794) apresentam os resultados de algumas avaliações que questionam "até que ponto muitos júris de cidadãos alcançaram a transparência do fornecimento das informações".

\subsection{Custo de um júri de cidadãos}

Os custos financeiros e de recursos humanos excessivos de planeamento, organização e realização de um JC representam outra questão crucial. Os custos perfazem cerca de $€ 30000$ (Lenaghan, 1999; Iredale e Longley, 2007), incluindo custos de recrutamento e a remuneração de jurados, testemunhas, moderadores, honorários do mediador, despesas de transporte, alojamento e refeições, e o custo do relatório final. o longo tempo de preparação e as despesas do pessoal envolvido nesta atividade também devem ser tidos em consideração. O custo total é extremamente elevado, o que é, claramente, um desincentivo. Face aos elevados recursos financeiros necessários e ao longo tempo de preparação (cerca de 5-6 meses), os estudiosos têm recomendado a adoção meramente ocasional deste método para serem discutidas questões complexas (New, 1998).

\section{Conclusões}

Segundo Fung e Wright (2001: 25), os esquemas deliberativos possuem três objetivos principais: a) atingir "fins públicos (...) mais eficazmente" do que outros instrumentos participativos; b) "gerar resultados justos e equitativos", graças à sua capacidade de concentração em problemas de pessoas desfavorecidas, proporcionando-lhes a oportunidade de participar nas decisões públicas; c) desenvolver uma prática mais ampla e aprofundada de participação, capaz de "envolver os cidadãos comuns". No entanto, tal como foi realçado por vários académicos (Gastil, 2000; Fung e Wright, 2001; Blondiaux e Sintomer, 2002; Chambers, 2003; Delli Carpini et al., 2004; Culyer e Lomas, 2006; Santos, 2005; Pateman, 2012), há o risco de uma lacuna entre a experiência empírica (o funcionamento real dos esquemas deliberativos) e o ideal de envolver os cidadãos em decisões coletivas.

Esta é a grande preocupação de muitos académicos, que acreditam que a avaliação rigorosa do mecanismo participativo é ainda uma área negligenciada na literatura sobre participação social, incluindo os métodos deliberativos (Abelson et al., 2003b; Lehoux et al., 2009).

28 A avaliação do exercício de participação dos cidadãos é uma necessidade ainda mais premente face à grande proliferação de novos mecanismos de participação nos últimos anos. Ainda existe muito pouco conhecimento sobre a sua qualidade e eficácia e sobre a possibilidade de diferentes métodos poderem atingir diferentes alvos incluídos no conceito de deliberação e participação pública. A mesma preocupação foi expressada por Culyer e Lomas (2006: 364): "Haverá alguma evidência científica de que os processos deliberativos funcionam verdadeiramente? A resposta imediata é nem por isso." Os autores defendem que a grande parte da literatura sobre processos deliberativos em sistemas de saúde "tem sido e continua a ser a defesa dos processos deliberativos, e não relatórios sobre a efetividade de processos bem definidos". Na verdade, apesar da vasta 
literatura sobre este assunto, ainda são poucos académicos que examinaram a efetividade dos métodos deliberativos na participação dos cidadãos em decisões que afetam a comunidade (Lehoux et al., 2009; Abelson et al., 2003a; Thurston et al., 2005). Tal como a OECD (2005) relata, ainda é observado um desequilíbrio entre a quantidade de tempo, recursos económicos e energia que os governos investem para envolver os cidadãos e a sociedade civil na tomada de decisões públicas e a quantidade de atenção que é dedicada à avaliação deste esforço. A necessidade de estudos que demonstrem a efetividade dos mecanismos participativos é quase vital para garantir a utilização adequada dos recursos públicos, incluindo o tempo e esforço dos cidadãos, que raramente são contabilizados nos custos da participação.

Deste modo, a abordagem deliberativa tem de ser levada a sério, sem pensar a priori que a prática corresponde ao discurso proclamado. Ainda estamos numa fase inicial da compreensão do modo como os processos deliberativos podem mudar opiniões, promover a tolerância e compreensão entre os grupos e capacitar as pessoas para deliberar. Certamente, os investigadores, decisores e profissionais podem ajudar a empreender estudos mais rigorosos nesta área.

\section{BIBLIOGRAFIA}

ABELSON, J., P. G. Forest, J. Eyles, P. Smith, E. Martin e F. P. Gauvin (2003a), “Deliberations about deliberative methods: issues in the design and evaluation of public participation process", Social Science and Medicine, 57, pp. 239-251.

ABELSON, J., J. Eyles, C. McLeod, P. Collins, C. McMullan, e P. G. Forest (2003b), “Does deliberation make a difference? Results from a citizens panel study of health goals priority setting", Health Policy, 66, pp. 95-106.

ARMOUR, A. (1995), “The citizens' jury model of public participation”, in: O. Renn, T. Webler e P. Wiedemann (eds.), Fairness and Competence in Citizen Participation, Boston, Kluwer Academic, pp. 57-69.

BENNETT, P. e S. Smith (2007), “Genetics, insurance and participation: How a Citizens' Jury reached its verdict", Social Science \& Medicine, 64, pp. 2487-98.

BLONDIAUX, L. e Y. Sintomer (2002), “L'impératif délibératif”, Politix, 15 (57), pp. 17-35.

CHAMBERS, S. (2003), "Deliberative Democratic Theory”, Annual Review of Political Science, 6, pp. 307-323.

COOTE, A. e J. Lenaghan (1997), Citizens's Juries: Theory into Practice, London, Institute for Public Policy Research - IPPR.

CROUCH, C. (1999), “La ampliación de la ciudadanía social y económica y la participación”, in S. García e S. Lukes (eds.), Ciudadanía, justicia social, identidad y participación, Madrid, Siglo Veintiuno, pp. 257-285.

CULYER, A. e J. Lomas (2006), "Deliberative processes and evidence-informed decision making in healthcare: do they work and how might we know?" Evidence \& Policy, 2 (3), pp. 357-71. 
DALE, M., B. Hanley, G. Needham, G. e J. Nicholson (1998), "Managing the organizational context for a citizens' jury", in S. Davies, S. Elizabeth, B. Hanley, B. New e B. Sang (eds.), Ordinary Wisdom. Reflections on an experience in citizenship and health, London, King's Fund, pp. 31-42.

DELAP, C. (2001), “Citizens' juries: reflections on the UK experience”, PLA Notes, 40: 39-42.

DELLI CARPINI, M., C. F. Lomax e J. Lawrence (2004), "Public Deliberation, Discursive Participation, and Citizen Engagement: A review of the Empirical Literature", Annual Review of Political Science, 7: pp. 314-344.

DENG, C.-Y. e C.-L. Wu (2010), “An innovative participatory method for newly democratic societies: The «civic group forum» on national health insurance reform in Taiwan", Social Science \& Medicine, 70: pp. 896-903.

DRYZEK, J. (2000), Deliberative Democracy and Beyond. Liberals, Critics, Contestations, Oxford, Oxford University Press.

DUNKERLEY, D. e P. Glasner (1998), “Empowering the public? Citizens' juries and the new genetic technologies". Critical Public Health, 8 (3), pp. 181-192.

ELSTER, J. (1998), Deliberative Democracy, Cambridge, Cambridge University Press.

ELWOOD, P. e M. Longley (2010), “My Health: Whose responsibility? A jury decides”. Journal of Epidemiology and Community Health, 64 (9), pp. 761-64.

FISHKIN, J. (1995), The Voice of the People. Public Opinion and democracy, New Haven, Yale University Press.

FONT, J. e I. Blanco (2007), "Procedural legitimacy and political trust: The case of citizen juries in Spain”, European Journal of Political Research, 46: pp. 557-589.

FUNG, A. e O. Wright (2001), "Deepening Democracy: Innovations in Empowered Participatory Governance", Politics \& Society, 29 (1), pp. 5-41.

GASTIL, J. (2000), “Is Face-to-Face Deliberation a Luxury or a Necessity?”. Political Communication, 17 (4), pp. 357-71.

GLASNER, P. (2001), "Rights or rituals? Why juries can do more harm than good", PLA Notes, 40: pp. 43-45.

GOOBERMAN-HILL, R., J. Horwood, J. e M. Calnan, (2008), “Citizens' juries in planning research priorities: process, engagement and outcome”, Health Expectation, 11, pp. 272-81.

HABERMAS, J. (1997), The theory of communicative action, Voll. 1 and 2, Boston, Beacon Press.

HABERMAS, J. (1992), "Further reflections on the Public Sphere”, in: C. Calhoun (ed.), Habermas and the Public Sphere, Cambridge, Massachusetts, The MIT Press.

HERBISON, P., J. Hay-Smith, H. Paterson, G. Elis e D. Wilson (2009), "Research priorities in urinary incontinence: Results from citizens' juries”, BJOG: An International Journal of Obstretrics and Ginecology, 116 (5), pp. 713-18.

IREDALE, R. e M. Longley (2007), “From passive subject to active agent: The potential of Citizens' Juries for nursing research", Nurse Education Today, 27, pp. 788-95.

KASHEFI, E. e M. Mort (2004), “Grounded citizens' juries: a tool for health activism?”, Health Expectations, 7, pp. 290-302. 
LEHOUX, P., G. Daudelin, O. Demers-Payette e A. Boivin (2009), "Fostering deliberations about health innovation: What do we want to know from publics?", Social Science \& Medicine, 68, pp. 2002-2009.

LENAGHAN, J. (1999), “Involving the public in rationing decisions. The experience of citizens' juries", Health Policy, 49, pp. 45-61.

LENAGHAN, J., B. New e E. Mitchell (1996), “Setting priorities: is there a role for citizens' juries?”, British Medical Journal, June, 312, pp. 1591-1593.

LOMAS, J. (1997), "Devolving authority for health care in Canada's provinces”, Canadian Medical Association, 156 (6), pp. 817-823.

MCIVER, S. (1998), Healthy Debate? An independent evaluation of citizens' juries in health settings, London, King's Fund.

MENON, D. e T. Stafinsky (2008), “Engaging the public in priority-setting for health technology assessment: Findings from a citizens' jury”, Health Expectations, 11 (3), pp. 282-93.

MITTON, C., N. Smith, S. Peacock, B. Evoy, B. e J. Abelson (2009), "Public participation in health care priority setting: A scoping review", Health Policy, 91, pp. 219-228.

MOONEY, G. e S. Blackwell (2004), "Whose health service is away? Community values in health care", Medical Journal of Australia, 180 (2), pp. 76-78.

NEW, B. (1998), “Citizens' juries: empowerment, self-development, informed view or arbitration?", in S. Davies, S. Elizabeth, B. Hanley, B. New e B. Sang (eds.), Ordinary Wisdom. Reflections on an experience in citizenship and health, London, King's Fund, pp. 92-115.

OECD - Organization for Economic Co-operation and Development (2005), Evaluating public participation in policy-making, Paris, OECD Publications.

PARKIN, L. e C. Paul (2011), “Public good, personal privacy: A citizens' deliberation about using medical information for pharmacoepidemiological research", Journal of Epidemiology and Community Health, 65 (2), pp. 150-56.

PARKINSON, J. (2004), "Why deliberate? The encounter between deliberation and new public managers", Public Administration, 82 (2), pp. 377-395.

PATEMAN, C. (2012), "Participatory Democracy Revisited”, Perspectives on Politics, 10 (1), pp. 7-19.

PAUL, C., R. Nicholls, P. Priest, R. McGee (2008), “Making policy decisions about population screening for breast cancer: The role of citizens' deliberation”, Health Policy, 85, pp. 314-320.

PICKARD, S. (1998), “Citizenship and Consumerism in Health Care: A critique of Citizens' Juries”, Social Policy \& Administration, 32 (3), pp. 226-244.

PRICE, D. (2000), “Choices without reasons: citizens' juries and policy evaluation”, Journal of Medical Ethics, 26, pp. 272-276.

SANTOS, B. S. (ed.)(2005), Democratizing Democracy. Beyond the Liberal Democratic Canon, London, Verso.

SCUTCHFIELD, F. D., L. Hall e C. L. Ireson (2006), “The public and public health organizations: Issues for community engagement in public health", Health Policy, 77, pp. 76-85.

SERAPIONI, M. (2002), "Forme innovative di partecipazione dei cittadini alla valutazione di qualità: Citizens' Juries e Comitati Consultivi Misti”, in C. Cipolla, G. Giarelli e L. Altieri (eds.), Valutare la qualità in sanità, Milan, Angeli, pp. 217-251. 
SERAPIONI, M. e N. Duxbury (2012), “Citizens' participation in the Italian health-care system: the experience of the Mixed Advisory Committees", Health Expectations (Online version, 19 Abril

2012). DOI: 10.1111/j.1369-7625.2012.00775.x.

SMITH, G. e C. Wales (2000), “Citizens' Juries and Deliberative Democracy”, Political Studies, 48, pp. 51-65.

TAYLOR, D. (1989), “Citizenship and social power”, Critical Social Policy, 9, pp. 19-31.

THURSTON, W. E., G. MacKean, A. Vollman, A. Casebeer, M. Weber, B. Maloff e J. Bader (2005), "Public participation in regional health policy: a theoretical framework", Health Policy, 73, pp. 237-252.

TIMOTIJEVIC, L. e M. Raats (2007), "Evaluation of two methods of deliberative participation of older people in food-policy development”, Health Policy, 82, pp. 302-319.

WAKEFORD, T. (2002), “Citizens Juries: a radical alternative for social research”, Social Research Update, 37, pp. 1-5.

ZAKUS, D. e C. Lysack (1998), “Revisiting community participation”, Health Policy and Planning, 13 (1), pp. 1-12.

\section{RESUMOS}

Nos últimos anos, as abordagens deliberativas receberam grande interesse dadas as suas potencialidades em melhorar a interação entre decisores e cidadãos. Um processo deliberativo caracteriza-se pela cuidadosa análise e discussão que empreende, de preferência em pequenos grupos, sobre as vantagens e desvantagens das possibilidades em debate, a definição de critérios de avaliação e o uso de tais critérios para selecionar a melhor solução. O debate sobre as abordagens deliberativas tem sido muito desenvolvido no setor da saúde, onde há uma crescente compreensão da necessidade de criar uma esfera pública apropriada para incentivar o diálogo entre os diferentes atores. Os Júris de Cidadãos têm sido o método deliberativo mais comum adotado nos processos deliberativos sobre saúde. Este artigo começa por debater a importância das abordagens deliberativas no setor da saúde para depois avaliar com mais detalhe o método do júri de cidadãos, realçando as suas principais características e evidenciando os seus pontos fortes e as suas debilidades.

In recent years, deliberative approaches have received much interest as an innovative strategy to enhance the interaction between decision-makers and citizens. A deliberative process is characterized by the careful examination and discussion - preferably in small groups (face-toface discussion) - of the advantages and disadvantages of the different options, the establishment of evaluative criteria and the use of these criteria for selecting the best solution. The debate on deliberative approaches is well developed in the health sector where there is a growing understanding of the need to create an appropriate public sphere to encourage the dialogue between the different actors in the health system. Citizens' Jury is the most common deliberative method adopted in the health systems. This article presents the debate on the importance of deliberative approaches in the health sector and, subsequently, describes the citizen's juries, highlighting its features, strengths and weaknesses. 
ÍNDICE

Keywords: health system, citizens participation, deliberative approaches, citizens' juries Palavras-chave: sistema de saúde, participação cidadã, abordagens deliberativas, júri de cidadãos

AUTOR

MAURO SERAPIONI

Centro de Estudos Sociais, Universidade de Coimbra (Portugal) (mauroserapioni@ces.uc.pt) 